\title{
Magnitude of water reward in the runway: A parametric investigation
}

\author{
PATRICK E. CAMPBELL and BRIAN M. KRUGER \\ Wright State University, Dayton, Ohio 45435
}

\begin{abstract}
Forty rats were given 42 acquisition and 15 extinction trials in a runway at 1 trial/day. The magnitude of water reward during acquisition was varied across five levels for independent groups $(.1,1.1,2.1,3.1$, and $4.1 \mathrm{ml})$. Acquisition response speeds were an inverted-U-shaped function of the magnitude of reward. The results of the extinction phase showed that the rats that received the smallest reward during training showed the greatest persistence during extinction. The extinction performance of the rats that received the larger rewards during training did not differ. The results of both the acquisition and extinction phases were seen as generally consistent with the literature on the magnitude of food reward.
\end{abstract}

Since the early experiments of Crespi(1942), Grindley (1929), and Zeaman (1949), there has been general agreement that instrumental performance is an increasing negatively accelerated function of the amount of reward. This generalization, although sometimes stated without reservations, is generally understood to apply (with some exceptions) to a small sample of species (rats, pigeons, and monkeys), to a restricted set of responses (running and leverpressing), and to a limited variety of rewards (dry food and liquid sucrose concentrations).

One purpose of the present experiment was to examine whether the generalization could be extended to include water reinforcement. Our interest in the variable of magnitude of water reward was stimulated by the suggestion of Macdonald and de Toledo (1974) that the mechanisms controlling persistent responding were different for water reward and for food reward. They reported the common partial reinforcement extinction effect (PRE) with food reward (one 45-mg food pellet) but failed to observe a PRE with water reward $(.1 \mathrm{ml})$. Experiments were soon published, however, demonstrating that a PRE could be obtained with water and that the extent of the PRE was an increasing function of the magnitude of water reward (Campbell, Hinson, \& Kruger, 1977; Seybert, Gerard, Lawrence, Nash, \& Williams, 1976). Although Seybert et al. and Campbell et al. independently selected similar amounts to represent "small," "medium," and "large" rewards (Seybert et al. selected .1, .8, and $1.6 \mathrm{ml}$; Campbell et al. selected $.1, .4$, and $.8 \mathrm{ml}$ ), the reward magnitude literature did not provide much guidance concerning the parametric range of water rewards.

Kintsch (1962) appears to have published the only study that varied the magnitude of water reward prior

The authors thank Jean Burdeshaw for running the animals for this experiment. Requests for reprints should be sent to Patrick E. Campbell, Department of Psychology, Wright State University, Dayton, Ohio 45435. to Seybert et al. (1976) and Campbell et al. (1977). Kintsch used $.25,1.75$, and $3.5 \mathrm{ml}$ of water as a reward for running a straight alley. The levels of water reward were combined factorially with three levels of thirst motivation in a balanced design. Kintsch reported a generally monotonic increase in running speed with increasing reward but only when thirst motivation was at its highest level. With the lowest drive level, the magnitude of water reward was irrelevant; with medium drive, only the largest reward magnitude resulted in faster running. Both Seybert et al. (23-1/2 h) and Campbell et al. (23-2/3 h) used more stringent deprivation procedures than Kintsch's highest level $(23 \mathrm{~h})$. Campbell et al. found a small increase in running speed with larger reward magnitudes; however, Seybert et al. observed no effect of reward magnitude on running speed during acquisition.

With respect to extinction performance following training with consistent reinforcement, both Seybert et al. (1976) and Campbell et al. (1977) reported that persistence was inversely related to the magnitude of training reward. Kintsch (1962), however, reported that extinction performance was unrelated to the magnitude of training reward, except perhaps for a temporary carry-over from terminal acquisition differences.

The present experiment was designed as a parametric study with a sufficient range of reward levels to cover the expected range of functional water-reward magnitudes (i.e., $.1 \mathrm{ml}$ to $4.1 \mathrm{ml}$ ). Only one trial was given each day to control for potential differences in withinsession satiation. Both Kintsch and Campbell et al. used a 1 trial/day procedure. Seybert et al.'s failure to observe differences in acquisition performance might have been caused by their use of multiple-trial sessions.

\section{METHOD}

Subjects
Forty naive female rats of the Sprague-Dawley strain were
purchased from Blue-Spruce Farms, Altamont, New York. The 
animals weighed approximately $180 \mathrm{~g}$ at the beginning of the experiment. They were housed in individual cages in a room with a 12-h-off, 12-h-on light-dark cycle. All sessions were conducted during the dark phases of the cycle.

\section{Apparatus}

The $160 \times 13 \times 9 \mathrm{~cm}$ straight runway and $37 \times 13 \times 9 \mathrm{~cm}$ goalbox were painted flat medium gray throughtout and covered with clear plastic lids. The 4-cm-long goal area of the goalbox contained a water cup and was separated from the 33-cm-long confinement area by a guillotine door. The confinement area was separated from the runway by a second guillotine door. Three water cups were used. For the smallest reward $(.1 \mathrm{ml})$, the cup measured $13 \mathrm{~mm}$ in diameter and $6 \mathrm{~mm}$ in depth. A $19 \times 9 \mathrm{~mm}$ cup was used for the 1.1- and 2.1-ml rewards, and a $23 \times 13 \mathrm{~mm}$ cup was used for the 3.1- and $4.1-\mathrm{ml}$ rewards. The cups had slightly rounded bottoms to permit the animal easy access to the full measure of water. The water was measured using a Labindustries Repipet calibrated to $.1 \mathrm{ml}$. A system of photoelectric relays and .01-sec clocks measured start, run, and goal times for consecutive 25-, 100-, and $25-\mathrm{cm}$ distances, respectively. The first infrared beam was located $25 \mathrm{~cm}$ from the start end of the alley, and the last was $20 \mathrm{~cm}$ inside the confinement area. Two $25-\mathrm{W}$ red lamps located $1 \mathrm{~m}$ above the goal area provided the illumination, and a 75-dB (SPL) white masking noise was present during sessions.

\section{Procedure}

Upon arrival, the rats were given free access to food and water for 7 days. Thereafter, the animals were maintained on a 23-2/3-h water deprivation schedule, but food was always available in the home cages. During each of the 6 days of pretraining, the animals were placed in groups of three on a table top. The reward cups described above were filled and placed on the table, and the animals were allowed to drink from them. In addition, each animal was handled for approximately 2-3 min during each day of the table-top training. On Days 1-2 the rats were also placed into the alley in groups of three for $5 \mathrm{~min}$. The water cup was full. On Days 3-4 the animals were individually placed directly into the goalbox and confined there for 3-5 min or until the animal drank from the goal cup. The procedure on Days 5-6 was the same as on Days 3-4, except that the animals were allowed to explore the runway before being transferred into the baited goalbox.

During the acquisition phase, one trial a day was given for 42 days. The animals were assigned at random to five groups and given $.1,1.1,2.1,3.1$, or $4.1 \mathrm{ml}$ of water reward on every trial. After entering the goalbox, the animals in these groups were removed after $30,30,60,90$, or $120 \mathrm{sec}$, respectively, or immediately after consuming the water if drinking time was less than the criterion time. Extinction trials were given for 15 days at one trial a day. The goalbox confinement time during extinction was $1 \mathrm{~min}$ for all animals.

Trials were begun by placing the rat in the starting area prior to the first infrared beam. On all trials, goalbox confinement began and the door to the goal opened when the rat interrupted the beam in the goalbox. Rats not traversing a section of the runway within $30 \mathrm{sec}$ were placed in the goalbox, and a time of $30 \mathrm{sec}$ was recorded for each section not traversed. All of the animals in a given reward magnitude group were run consecutively, but the order of running the groups was determined randomly each day.

\section{RESULTS}

The response times for each alley segment were converted to speeds (centimeters/second) prior to analysis. The acquisition response speeds for each of the reward groups were averaged over blocks of six trials and are shown in Figure 1. An inspection of the figure suggests that running speeds increased with larger rewards, with the exception of the largest reward group. In the run and goal sections, the animals receiving 4.1-ml rewards ran no faster than the animals receiving only $.1 \mathrm{ml}$. In the start section, the $4.1-\mathrm{ml}$ rats eventually ran somewhat faster than the $.1-\mathrm{ml}$ rats but continued to run slower than the other reward groups. Analyses of variance confirmed a significant $(p<.05)$ reward magnitude effect in each alley measure $[\mathrm{F}(4,35)=6.17$ for start, $F(4,35)=4.44$ for run, and $F(4,35)=3.50$ for goal]. In addition, a significant $(\mathrm{p}<.05)$ Reward Magnitude by Trial Blocks interaction was found for each alley section $[F(24,210)=2.13$ for start, $F(24,210)$ $=2.15$ for run, and $F(24,210)=1.63$ for goal]. Trend analyses of the reward magnitude main effects showed significant $(p<.05)$ quadratic components in each alley measure $[F(1,35)=20.76$ for start, $F(1,35)=$ 13.72 for run, and $F(1,35)=12.65$ for goal]. The quadratic trends accounted for $84 \%, 77 \%$, and $90 \%$ of the reward magnitude variance for each alley section,
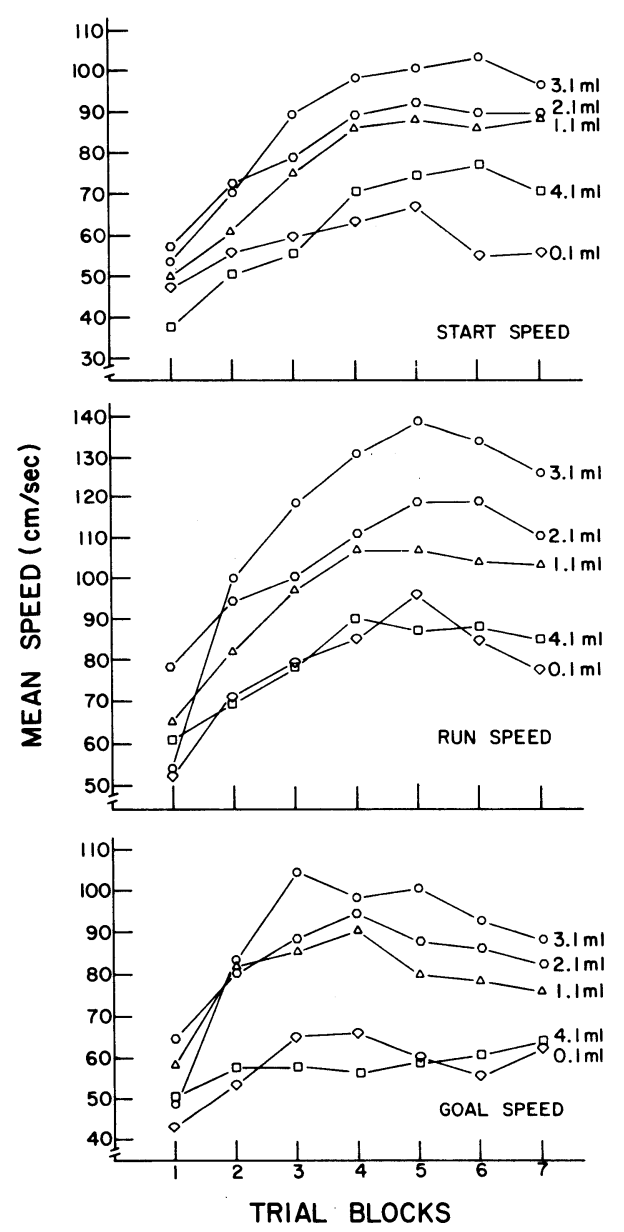

Figure 1. Mean response speeds for each reward group in blocks of 6 days. 

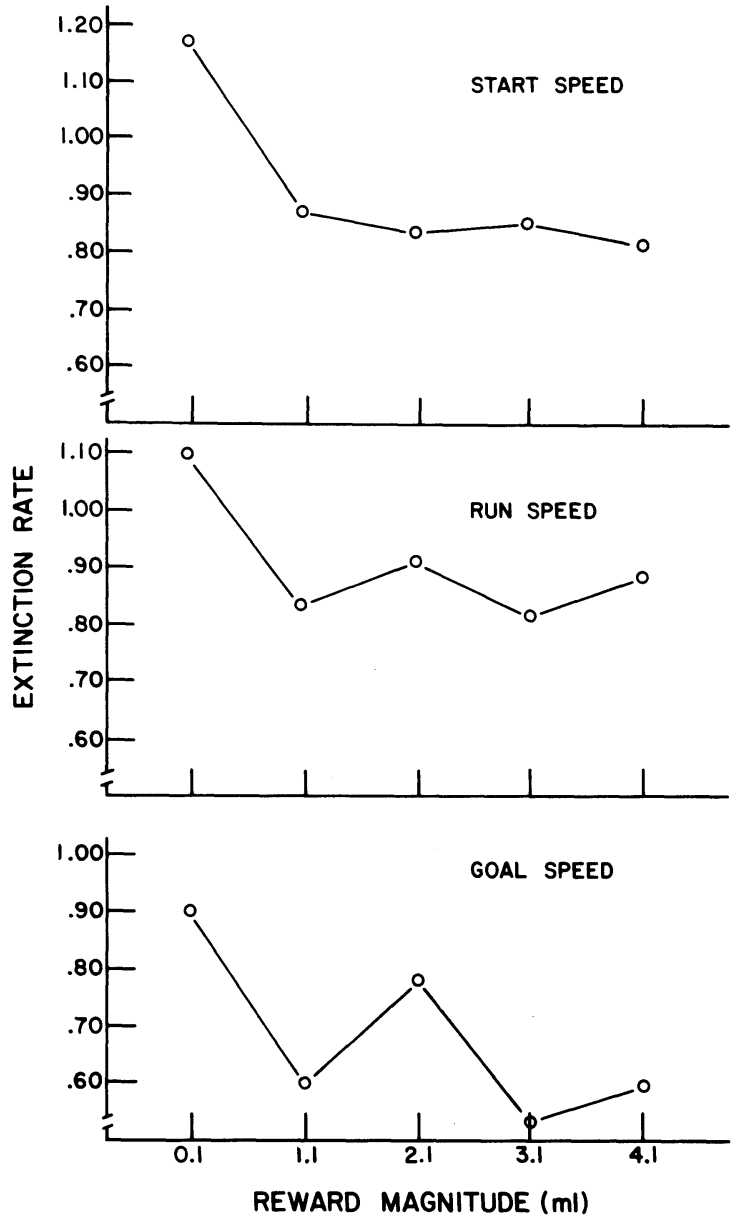

Figure 2. Mean rate of extinction scores based on speeds for each reward magnitude group.

respectively. None of the other trend components approached significance in any alley section.

Since considerable differences in response speeds were present at the end of the acquisition phase, the extinction-speed data were converted to a rate measure following the suggestions outlined in Anderson (1963, Equation 4). The speed score of each animal during the last block of acquisition training served as an estimate of acquisition response levels, and the minimum response speed allowed during extinction was used as the extinction estimate. These rate scores, averaged over all 15 extinction trials, are shown in Figure 2. These data suggest that extinction persistence was greatest for the smallest reward animals and that the difference among the four large-reward groups was negligible. Analyses of variance showed significant $(\mathrm{p}<.05)$ reward magnitude effects for the start $[F(4,35)=4.94]$ and goal sections $[F(4,35)=3.14]$. The main effect in the run section approached significance $(\mathrm{F}=2.25, .05<\mathrm{p}<.10)$.

\section{DISCUSSION}

The results of the present study suggest that acquisition performance in the runway is an inverted-U-shaped function of the magnitude of water reward. With the exception of the largest reward group $(4.1 \mathrm{ml})$, the present results are consistent with the commonly accepted generalization that performance is an increasing negatively accelerated function of the magnitude of reward. Roberts (1969) reported a parametric investigation with a wide sampling of reward levels that can be considered as representative of the general literature dealing with the magnitude of food reward. Roberts trained five groups of rats for $\mathbf{4 8}$ trials at $1 \mathrm{trial} / \mathrm{day}$. The groups received $1,2,5,10$, or 25 pellets (Noyes $45 \mathrm{mg}$ ). Asymptotic speeds indicated monotonic negatively accelerated curves in the run and goal sections of the alley. In the start section, the speed curve was S-shaped, with little or no improvements in performance beyond the fivepellet reward.

The present results are generally consistent with the food reinforcement literature over most of the range of water rewards sampled. Our largest reward group, however, clearly contradicts the monotonic function usually reported with food reward. Nevertheless, we are not willing to suggest that the functions relating food reward and water reward to running speeds are fundamentally different. It is possible that a wider sampling of food rewards (Roberts sampled up to a $25: 1$ ratio) would result in an inverted-U function similar to the function observed in the present study (we sampled up to a $41: 1$ ratio).

A second common generalization derived from the reward magnitude literature is that the performance curves for different amounts of reward eventually reach different asymptotic levels but that the rates of approach to the separate asymptotes are constant. Recent evidence, however, suggests that the above generalization may be true only because very few studies continue acquisition beyond the point where apparent asymptotic performance is reached. McCain, Dyleski, and McElvain (1971) compared large (one 500-mg pellet) and small (one $45-\mathrm{mg}$ pellet) reward in a runway in seven studies that varied the amount of training $(24,54,60,70,78,90,116$, or 135 trials). They reported that large reward led to faster running after limited training ( 24 trials) but that the effect of reward magnitude was minimal or nonexistent after extended training (54-135 trials). Campbell, Batsche, and Batsche (1972) trained rats for 120 daily trials with small or large food rewards delivered in either single-pellet $(97 \mathrm{mg}$ vs. $1,000 \mathrm{mg})$ or multiple-pellet $(2-45 \mathrm{mg}$ vs. $22-45 \mathrm{mg})$ form. In the multiple-pellet condition, the large-reward rats maintained faster speeds than the smallreward rats for approximately 90 trials, but by the end of training, the two groups did not differ. When the amount of reward was varied by the weight of a single pellet, the large-reward rats ran faster in the start section for approximately 70 trials but never exceeded the performance of the small-reward rats in the run and goal sections. By the end of training, the smallreward rats were running faster than the large-reward rats in all alley sections.

Our original intention was to continue the present study beyond 42 acquisition days. Unfortunately, several animals developed bladder infections during the later stages of training. Since extinction performance was a part of our experimental plan, we elected to begin extinction after only 42 training trials. Nevertheless, an inspection of our speed curves suggests a trend that is consistent with the results of McCain et al. (1971) and Campbell et al. (1972). Maximum speeds were observed in the start measure by Block 6 , in the run measure by Block 5 , and in the goal measure by Block 4 . In the run and goal sections, where maximum speeds occurred relatively early, there was a general decline in response speeds with continued training for the three fastest groups. These observations suggest that, with a longer training period, the results using water reinforcement might prove to be consistent with the extended training food studies reported by McCain et al. and Campbell et al.

With respect to the extinction measure, the present results can be described rather simply. The smallest reward group showed greater persistence than the larger reward groups, and 
the larger reward groups did not differ among themselves. The Campbell et al. (1977) data, however, showed a decreasing monotonic function when $.1, .4$, and $.8 \mathrm{ml}$ were sampled. Seybert et al. (1976) reported a decreasing function, but, like the present results, their larger reward groups did not differ in extinction (they sampled $.1, .8$, and $1.6 \mathrm{ml}$ ). When the results of all three studies are considered together, it appears that extinction persistence is a decreasing negatively accelerated function of the magnitude of water reward; the function is relatively steep, reaching its maximum at approximately $.8 \mathrm{ml}$. Kintsch (1962) reported that the extinction performance of the reward magnitude groups was similar to the terminal acquisition differences but that the initial differences soon disappeared. Since Kintsch did not publish extinction curves or attempt to adjust the extinction scores for terminal acquisition levels, it is not possible to judge whether his subjects might have differed in terms of extinction rates.

The generalization that extinction persistence is a negatively accelerated decreasing function of the magnitude of water reward is consistent with the literature on food reward. Again, the Roberts (1969) study can be considered to be representative of the recent food literature. He reported a negatively accelerated decreasing function in each alley measure, but the steepness of the function was clearly influenced by which alley measure was considered. In the start measure, there was a clear separation among all of the groups. In the run and goal sections, the function seemed to reach its maximum at five pellets, with little or no separation among the larger reward groups.

With both food and water reward, then, it appears that the functionally effective range of reward magnitudes is large when acquisition performance is considered and much smaller when extinction is the process of interest. Although the number of published studies that have varied the magnitude of water reward is quite small, these studies are generally consistent with the much larger literature on the magnitude of food reward.

Although the present discussion was limited to generalizations concerning consistent reinforcement schedules, both Campbell et al. (1977) and Seybert et al. (1976) reported other similarities between food and water reinforcement within the context of partial reinforcement schedules.

\section{REFERENCES}

Anderson, N. H. Comparisons of different populations: Resistance to extinction and transfer. Psychological Review, 1963, 70, 162-179.

Campbell, P. E., Batsche, C. J., \& Batsche, G. M. Spacedtrials reward magnitude effects in the rat: Single versus multiple food pellets. Journal of Comparative and Physiological Psychology, 1972, 81, 360-364.

Campbell, P. E., Hinson, T. A., \& Kruger, B. M. Runway performance as a function of the schedule and magnitude of water reward. Bulletin of the Psychonomic Society, 1977, 10, 69-72.

CresPI, L. P. Quantitative variation of incentive and performance in the white rat. American Journal of Psychology, 1942, 55, 467-517.

Grindley, G. C. Experiments on the influence of the amount of reward on learning in young chickens. British Journal of Psychology, 1929, 30, 173-180.

KINTSCH, W. Runway performance as a function of drive strength and magnitude of reinforcement. Journal of Comparative and Physiological Psychology, 1962, 55, 882-887.

Macdonald, G. E., \& DE Toledo, L. Partial reinforcement effects and type of reward. Learning and Motivation, 1974, 5, 288-298.

McCain, G., Dyleski, D., \& McElvain, G. Reward magnitude and instrumental responses: Consistent reward. Psychonomic Monograph Supplements, 1971, 3, 249-256.

Roberts, W. A. Resistance to extinction following partial and consistent reinforcement with varying magnitudes of reward. Journal of Comparative and Physiological Psychology, 1969, 67, 395-400.

Seybert, J. A., Gerard, I. C., Lawrence, W. T., Nash, S. W., \& Williams, C. L. Effects of schedule and magnitude of reinforcement under conditions of thirst motivation. Learning and Motivation, 1976, 7, 559-570.

ZEAMAN, D. Response latency as a function of the amount of reinforcement. Journal of Experimental Psychology, 1949, 39, 466-483.

(Received for publication June 4, 1979.) 